\title{
Dynamical origin of the asteroid (25143) Itokawa: the target of the sample-return Hayabusa space mission (Research Note)
}

\author{
P. Michel ${ }^{1}$ and M. Yoshikawa ${ }^{2}$ \\ 1 Observatoire de la Côte d'Azur, UMR 6202 Cassiopée/CNRS, BP 4229, 06304 Nice Cedex 4, France \\ e-mail: Patrick.Michel@obs-azur.fr \\ 2 Japan Aerospace Exploration Agency, Institute of Space and Astronautical Science, 3-1-1 Yoshinodai, Sagamihara, Kanagawa 229-8510, \\ Japan
}

Received 7 October 2005 / Accepted 22 December 2005

\section{ABSTRACT}

Aims. We have studied numerically the origin and dynamical evolution of the asteroid (25143) Itokawa on which a Japanese space probe landed and may have collected a sample. The return to Earth is planed in 2010.

Methods. To estimate the most probable source of Itokawa, we have used the most recent model of the Near-Earth Object population, which allows us to relate the orbital parameters of a NEO to the different identified source regions of the NEO population.

Results. The two source regions that are the most likely to transport an object to Itokawa's orbit are the $v_{6}$ secular resonance in the main belt and at a lower level of probability the Mars-crosser population. This result is consistent with the spectral characteristics of Itokowa, identified as S-type, which is the dominant spectral type of objects in these sources. In a previous study, we had found that its most likely fate may be an impact with the Earth on a million year timescale. Here, we show that its evolution is consistent with the typical evolution of asteroids coming from the identified source and which are extracted in the Earth-crossing zone by planetary encounters.

Conclusions. We conclude that Itokawa is a typical NEO, which probably arrived at its orbit from the main belt through the $v_{6}$ channel. It belongs to the most common spectral-type in the inner Solar System. Therefore, unless this asteroid had a peculiar collisional history, the data obtained by the satellite Hayabusa will clarify some properties representative of the S-type NEO population.

Key words. celestial mechanics - methods: $N$-body simulations - minor planets, asteroids - solar system: general

\section{Introduction}

We provide an estimate of the most likely source region of the asteroid Itokawa which was visited by the JAXA space mission Hayabusa during the period September-December 2005 (Fujiwara et al. 2000). Based on this estimate, the analysis of the data obtained by this mission will allow us to better characterize the properties of the population of small bodies evolving in this region, assuming that Itokawa is not a peculiar object.

The physical properties of main belt asteroids and NearEarth Objects (NEOs) still rely mostly on remote observations, as only a few asteroids have been visited by spacecrafts. Therefore, we still have a very poor knowledge of the properties of these small body populations which contain essential information on the original composition of the solar nebula. Moreover, in the framework of protection strategies, it is crucial to have an accurate knowledge of the properties of potentially hazardous objects in order to define appropriate methods to avoid an impact. The precise link between meteorites and asteroids will also be better established if we can compare asteroid samples with meteorites.
So far, no space mission has brought back a sample of a small body, and this was the purpose of the Hayabusa mission, originally named MUSES-C. The landing on the asteroid occured on November 26, 2005. Unfortunately, the success of the sampling procedure is not guaranted. The return to Earth, originally planed for June 2007, has been postponed to 2010 for technical reasons.

The determination of the origin of Itokawa is of great importance. Under the assumption that this asteroid is representative of other bodies in its source region, the analysis of its properties and of its sample, if collected, can provide for the first time a direct link between physical properties and a population of small bodies in a known location. However, the representativity of this object is difficult to assess. Spectral observations indicate that the asteroid belongs to the $\mathrm{S}$ taxonomic class, which is the dominant class in the NEO population and in the inner asteroid belt; its geometric albedo may be about 0.35 , which is higher than usual for S-type objects in general but which may correspond to usual values for kilometersized bodies (Delbo et al. 2003); its rotation period is about $12.13 \mathrm{~h}$, which is longer than average (close to $6 \mathrm{~h}$ for objects 
of this size). Based on this limited information, it is difficult to conclude whether it is a representative body. The first highresolution images taken by the Hayabusa probe showed that its shape is very irregular, probably composed of several rocks linked together by gravity, which may indicate that the object is a rubble pile. Its surface contains many boulders and small debris but only a few craters are seen.

The determination of the potential region of origin would indicate whether the asteroid comes from a location already known as a source of NEOs. Then, estimating its long-term dynamical evolution, we can analyse whether it is a dynamically peculiar object or if its behavior makes it a typical NEO. In a previous paper, the results of long term numerical integrations of Itokawa's orbital evolution were presented (Michel \& Yoshikawa 2005, hereafter MY 2005). We found that Itokawa's evolution is dominated by planetary close approaches, mainly with the Earth and Venus, and that it may collide with the Earth, in several million years. Its evolution is typical to that of most NEOs in the Earth-crossing region. We thus concluded that Itokawa is not a peculiar object and has the classical dynamical behavior of NEOs. Both the estimate of its origin and of its potential evolution can serve as a tool to determine whether Itokawa is a representative object or if it is strange in some respects. As we will show in the following, our results suggest that Itokawa is a representative object.

\section{Origin of the asteroid (25143) Itokawa}

We use the NEO model of Bottke et al. (2002a) to determine the most likely source region of Itokawa.

Bottke et al. (2000, 2002a) created a steady state model of the orbital and absolute magnitude $(H)$ distribution of the NEO population, which corresponds to a best fit of the debiased orbital and absolute magnitude distributions to the observed NEO population. The model relies on the existence of well-identified sources of NEOs. Several decades of dynamical studies have demonstrated that the most efficient sources of small bodies for NEO orbits are the 3:1 mean motion resonance with Jupiter, the $v_{6}$ secular resonance, the Mars-crossing asteroids (MC), the outer main belt at a semimajor axis $a>2.8 \mathrm{AU}$ (OB), and the Jupiter Family comets (JFC). The positions of these different source regions are indicated in Fig. 1. Two additional sources were known but were not included in the original model because their role was assumed to be negligible to a first order approximation. These two sources have since been taken into account (Bottke et al. 2004): the Hungarias (HU), characterized by a semimajor axis ranging from 1.77 to $2.06 \mathrm{AU}$ and an inclination greater than $15^{\circ}$, and the Phocaeas (PHO), characterized by a semimajor axis from 2.1 to $2.5 \mathrm{AU}$ and an inclination that place them above the $v_{6}$ resonance.

The real NEO orbital distribution and the relative importance of the identified NEO source regions to one another were estimated (see Bottke et al. 2002a, for details). The contributions of these sources have been updated recently by accounting for the remaining sources that were neglected in previous studies. The resulting distribution is consistent with the major NEO observational campaigns (e.g. Stuart \& Binzel 2004). The contribution factors indicate the relative importance of each

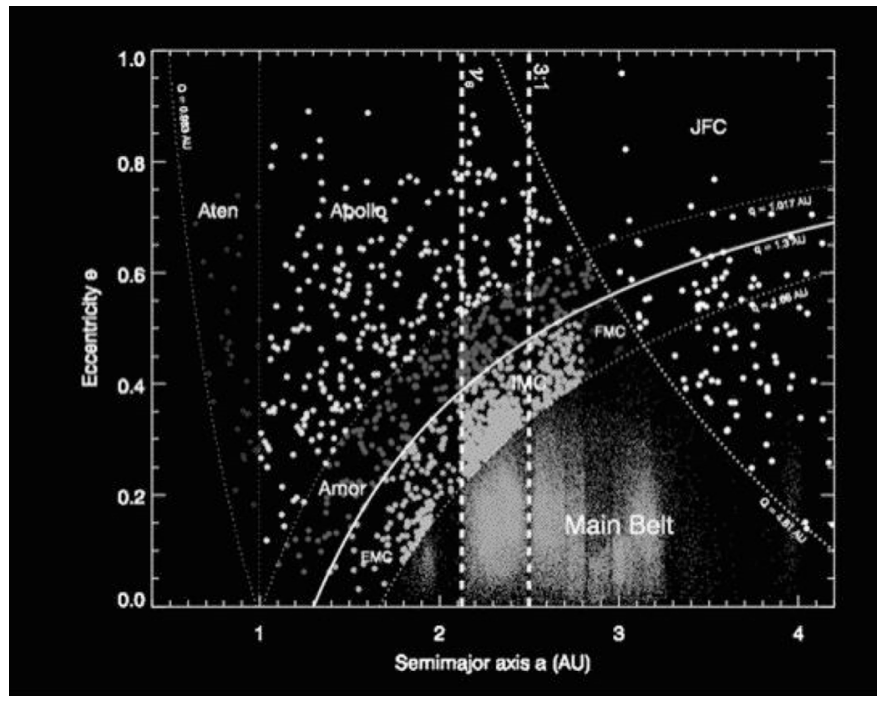

Fig. 1. Intermediate Source (IS) regions of the NEO population represented in the $(a, e)$ plane where $a$ is the semimajor axis and $e$ is the eccentricity. The three NEO groups, namely Atens, Apollos and Amors, and the Evolved Mars-Crossers (EMC, see Michel et al. 2000b) are also represented. The dots in different gray scales represent observed objects in the different regions. According to our estimate, Itokawa is likely to come from the main belt in the vicinity of the dotted-line labeled $v_{6}$ or from the region labeled IMC.

source to the whole NEO distribution. As a by-product they allow one to estimate which of these sources is the most likely to transport an object to a given orbital position. Thus, it is possibe to estimate the relative probability that a body on a given orbit $(a, e, i)$ in the NEO region, such as Itokawa, comes from a particular source.

To determine the most likely origin of Itokawa, we computed the relative probabilities $P_{1}=P_{3: 1}, P_{2}=P_{v_{6}}, P_{3}=P_{\mathrm{MC}}$, $P_{4}=P_{\mathrm{HU}}, P_{5}=P_{\mathrm{PHO}}, P_{6}=P_{\mathrm{OB}}, P_{7}=P_{\mathrm{JFC}}$ that an object with the orbital elements of Itokawa (refered to Ecliptic and Equinox 2000), $a=1.324 \mathrm{AU}, e=0.28$ and $i=1.623$ comes from the associated sources. Our computation gives $P_{1}=P_{5}=P_{6}=P_{7}=0$, with $P_{2}=0.64, P_{3}=0.35$ and $P_{4}=0.01$. Thus, according to this model, the probability that Itokawa originated from the main belt via the $v_{6}$ resonance is $64 \%$, whereas it has a $35 \%$ chance of coming from the Mars-crosser population and $1 \%$ chance of coming from the Hungaria region.

The most probable source region of Itokawa is the inner part of the main belt. This is fully consistent with the fact that Itokawa is an S-type asteroid, which is the dominant type in this region of the Solar System. Therefore, if this object is representative of the population of inner main belt asteroids, then important information on the properties of these asteroids will be provided by the data obtained by the satellite Hayabusa.

\section{Some aspects of the future orbital evolution of Itokawa}

The orbital evolutions of 39 Itokawa's orbits, obtained by slightly changing one initial orbital element at a time or by using different computers, under the gravitational perturbations 
of the planets and the Sun have been followed until they either collided with a planet or the Sun, or they were ejected outside Saturn's orbit. The reason for integrating so-called clone orbits in addition to the nominal one and the numerical integrator used to perform the integrations are described in MY (2005). These evolutions cover different parts of the orbital element space and indicate the possible dynamical mechanisms that are likely to affect the future evolution of Itokawa.

Although Itokawa's orbit is situated deep in the Earth-crossing region and is therefore extremely chaotic, its orbital evolution does not show drastic qualitative differences in the different integrations. All the evolutions are largely dominated by close approaches with the Earth and Venus, and have the typical behavior of a "classical" NEO evolution. As for most NEOs, the evolutions are charaterized by drastic semimajor axis changes as a result of planetary close encounters until a collision occurs, or until the semimajor axis reaches main belt values where it can encounter a powerful resonance (e.g. the $v_{6}$ secular resonance). In the last case, the eccentricity increases very rapidly until the body collides with the Sun or is ejected by a Jupiter encounter at its aphelion. Such evolutions are typical for NEOs and no dynamically peculiar behaviors have been found. Here, we show an example that is both representative of these evolutions and consistent with our result on its origin (Sect. 2).

Figure 2 (top) shows an example of the mechanisms that can send deep Earth-crossers into dangerous main belt regions. The body is first dominated by planetary close approaches, as indicated by the variations in semimajor axis. These variations lead to larger values of this parameter which eventually drive the body into the $v_{6}$ secular resonance (Fig. 2). While the resonant angle librates, the eccentricity goes very rapidly to 1 , which sends the asteroid into the Sun. If Itokawa originated from the main belt and was transported to its orbit via the $v_{6}$ resonance (see Sect. 2), then its history may correspond to one of the particle histories of Gladman et al. (1997, Fig. 3c). The authors computed the evolution of thousands of particles initially located in the main belt inside the $v_{6}$ resonance. Some particles are extracted from the resonance by an encounter with the Earth and evolve deep in the Earth-crossing zone before being sent back in the resonance and finally into the Sun. Starting from the current orbit of Itokawa, the evolution shown here can be interpreted as the last part of the evolution of an object coming from this source region.

In addition to gravity, another force has been found to contribute to the long term evolution of small asteroids and could induce objects in the size range $0.1 \mathrm{~m}-20 \mathrm{~km}$ to spiral inward or outward at different rates. This so-called Yarkovsky effect is a thermal radiation force that causes small bodies to undergo semimajor axis drift as a function of their size, spin, orbit and material properties (see, e.g. Bottke et al. 2002b). In our previous paper (MY 2005), we used the numerical integrator developed by Broz (2002), based on the swift package by Levison \& Duncan (1994), in which the Yarkovsky force is implemented. Appropriate material parameters for Itokawa required to compute the Yarkovsky effect were adopted. As expected, the possible evolution computed with this integrator did not result in drastically different orbital evolution from the purely

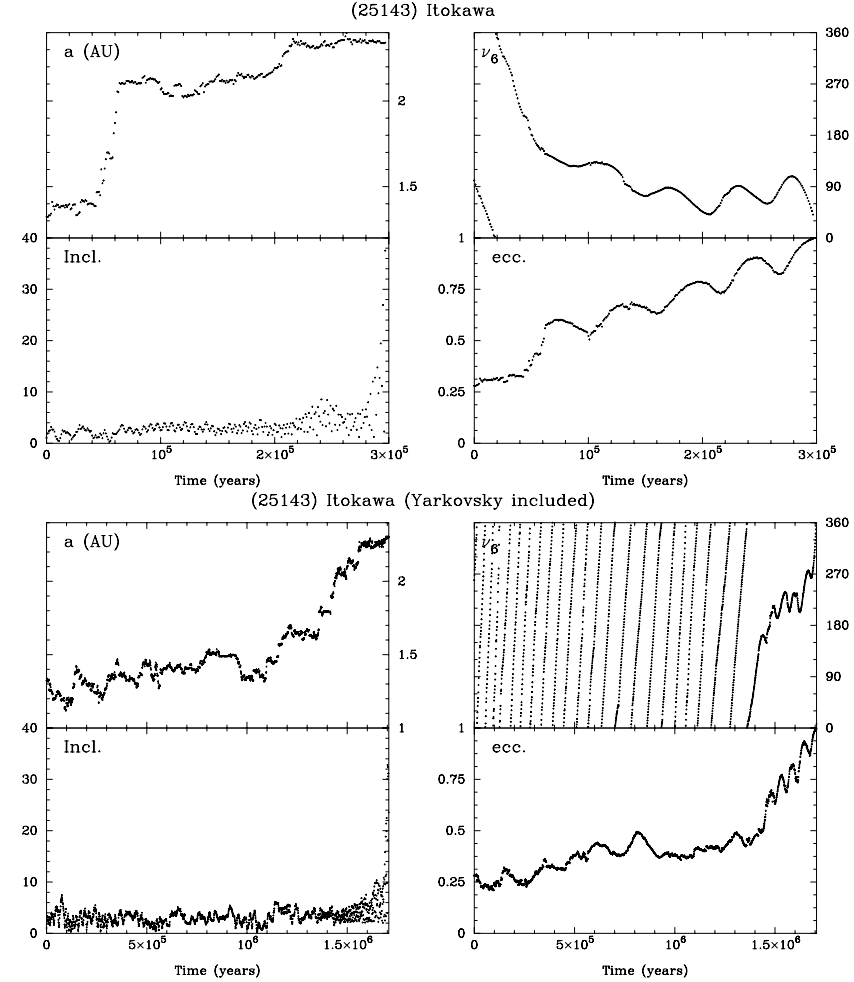

Fig. 2. Possible orbital evolution of Itokawa. Besides the semimajor axis (AU), inclination (degrees) and eccentricity, the figure shows the evolution of the critical argument of the $v_{6}$ resonance which is defined by $\left(\varpi-g_{6} \times t-\alpha_{6}\right)$, where $\varpi$ is the perihelion longitude of Itokawa's orbit, $g_{6}=28.22 \mathrm{arcsec} / \mathrm{yr}$ is the fundamental frequency corresponding to the resonance and $\alpha_{6}$ is the phase at time $t=0$. Top: purely gravitational evolution; bottom: evolution under gravitational perturbations and the Yarkovsky effect.

gravitational ones (see MY 2005). An example which may be related to the problem of Itokawa's origin is shown in Fig. 2 (bottom). The body follows the same kind of trajectory and has the same fate as in the absence of the Yarkovsky force. Therefore, we conjecture that although the Yarkovsky force may have acted while Itokawa belonged to the main belt - if it served to move it into the $v_{6}$ resonance - this force had a negligible role once the body was extracted from the resonance in the Earth-crossing zone.

\section{Conclusion}

We have estimated the origin of the asteroid Itokawa. Then, from the results of previous long term numerical integrations of its orbit, we showed that its possible future pathway is consistent with the typical evolution of bodies coming from the identified origin.

To estimate the source region of Itokawa, we used the model of the NEO population developed by Bottke et al. (2002) which indicates the contributions of the different sources of NEOs that lead to the complete (unbiased) orbital distribution of the NEO population. This model was recently refined to account for additional sources (Bottke et al. 2004). We found that Itokawa has a $64 \%$ probability of coming from the main belt via the $v_{6}$ resonance, while the probability that it comes from 
the Mars crosser population is around 35\%. A very small probability $(1 \%)$ is found that it comes from the asteroids of the Hungaria group and the remaining sources cannot place an object on Itokawa's orbit. Therefore, the origin of Itokawa probably lies in the inner asteroid main belt. This result is consistent with its $\mathrm{S}$ taxonomic type, which is the dominant type in this region of the Solar System.

Long term orbital projections of Itokawa (MY 2005) show dynamical behaviors consistent with the typical evolution of Earth-crossers coming from the main belt through the $v_{6}$ resonance (Gladman et al. 1997, Fig. 3c). Itokawa has the typical behavior of an Earth-crosser. It is strongly perturbed by close approaches, and it will probably spend some time in the region where Atens and the so-called Inner Earth Objects (IEOs; see Michel et al. 2000a) evolve. Based on the estimate of the collisional lifetime of Itokawa with the Earth (MY 2005), this body is likely to end its evolution by an Earth impact on a million year timescale.

We also checked that the Yarkovsky effect has no visible contribution to the long term evolution of the asteroid. However, the Yarkovsky force may still have played a dominant role by injecting Itokawa into the $v_{6}$ resonance during its main belt period. Indeed, if Itokawa was originally far from the resonance, the only way to reach the appropriate semimajor axis would be a semimajor axis drift caused by the Yarkovsky force. Another scenario could be that it belonged to the swarm of small fragments produced by the the disruption of a larger asteroid in the immediate vicinity of the resonance. Typical ejection velocities of fragments from large disruptions (see e.g. Michel et al. 2001) imply that the original parent body must have been close enough to the resonance to directly send some fragments into it. In that case, if Itokawa was recently sent to its current position, such a disruption should have left traces (such as an asteroid family). No such trace has been detected yet. However, based on the detailed images taken by Hayabusa, Itokawa looks like an aggregate, which may have been formed by the reaccumulation of smaller fragments as a result of a large body disruption (e.g. Michel et al. 2001). We will investigate this further once the complete set of data of the Hayabusa mission is available.

The visit of Itokawa by the Hayabusa space mission will shed some light on these important issues. Assuming the collisional history of Itokawa is as typical as its dynamical one, then we can conjecture that the properties of this asteroid measured during the visit of the satellite Hayabusa and, ideally, the samples that may be brought back, will be representative of the properties of the whole population of kilometer-size $\mathrm{S}$ type asteroids coming from the inner main belt. Such data will have implications for many fields of asteroid studies, such as establishing links between meteorites and asteroids or the original composition of the solar nebula or efficient mitigation strategies. Sample return space missions targeted to other asteroids with different taxonomic types are required to clarify the physical properties of the whole asteroid population.

Acknowledgements. We are grateful to $\mathrm{M}$. Abe for fruitful discussions concerning the physical properties of Itokawa, and Hajime Yano and the anonymous referee for their suggestions. We also thank A. Morbidelli and W. F. Bottke for the refined model of NEOs that they kindly provided, and A. Fujiwara from the Japanese JAXA/ISAS for financial support.

\section{References}

Bottke, W. F., Jedicke, R., Morbidelli, A., et al. 2000, Science, 288, 2190

Bottke, W. F., Morbidelli, A., Jedicke, R., et al. 2002a, Icarus, 156, 399

Bottke, W. F., Vokrouhlický, D., Rubincam, D. P., et al. 2002b, in Asteroid III, ed. W. F. Bottke, A. Cellino, P. Paolicchi, \& R. Binzel (Tucson: University of Arizona Press), 395

Bottke, W. F., Morbidelli, A., Jedicke, R., et al. 2004, AAS/Division of Planetary Sciences Meeting Abstracts, 36

Broz, M. 2002, Paper presented at Asteroids, Comets, Meteorss, Berlin, Germany, 29 July-2 August 2002

Delbó, M., Harris, A. W., Binzel, R. P., et al. 2003, Icarus, 166, 166

Fujiwara, A., Mukai, T., Kawaguchi, J., \& Uesugi, K. T. 2000, Adv. Space Res., 25 (2), 231

Gladman, B. J., Migliorini, M., Morbidelli, A., et al. 1997, Science, 277, 197

Levison, H. F., \& Duncan, M. J. 1994, Icarus, 108, 18

Michel, P., Zappalà, V., Cellino, A., \& Tanga, P. 2000a, Icarus, 143, 421

Michel, P., Migliorini, F., Morbidelli, A., \& Zappalà, V. 2000b, Icarus, 145,332

Michel, P., Benz, W., Tanga, P., \& Richardson, D. C. 2001, Science, 294, 1696

Michel, P., \& Yoshikawa, M. 2005, Icarus, 179, 291

Stuart, J. S., \& Binzel, R. P. 2004, Icarus, 170, 295 